\title{
Geometric Phase Metasurface Hologram for Optical Tractor Beam Generation
}

\author{
Jasper Cadusch*1, Dandan Wen ${ }^{1}$, Jiajun Meng ${ }^{1}$ and Kenneth B. Crozier ${ }^{* 1,2}$ \\ ${ }^{1}$ Department of Electrical and Electronic Engineering, University of Melbourne, Victoria 3010, Australia \\ ${ }^{2}$ School of Physics, University of Melbourne, Victoria 3010, Australia \\ Authore-mail address:*caduschj@unimelb.edu.au, kenneth.crozier@unimelb.edu.au
}

\begin{abstract}
We present a geometric phase silicon metasurface hologram design intended to produce a non-diffracting solenoid beam. Such optical beams have been shown to exert long range retrograde (i.e. toward source) optical forces on light-scattering particles. (C) 2020 The Authors
\end{abstract}

\section{Introduction}

Recent progress in holographic optical tweezers has included the development of optical tractor and conveyer beams [1]. These translational quasi-invariant beams allow for long-range, retrograde (i.e. toward source) optical forces to be exerted on microscopic particles. A particularly promising class of non-diffracting beams is the solenoid beam, created via a coaxial superposition of Bessel beams of different order. Typically, a solenoid beam is generated by adding a phase profile to a Gaussian beam with a spatial light modulator (SLM) to convert it into a helical beam carrying orbital angular momentum (OAM). The use of an SLM as a phase mask is costly and requires the use of bulk optics, including carefully aligned lenses. Here we present an alternative method based on a silicon geometric phase metasurface hologram that will produce a tripe helix solenoid beam at a wavelength of $1064 \mathrm{~nm}$.

\section{Calculation Results and Discussion}

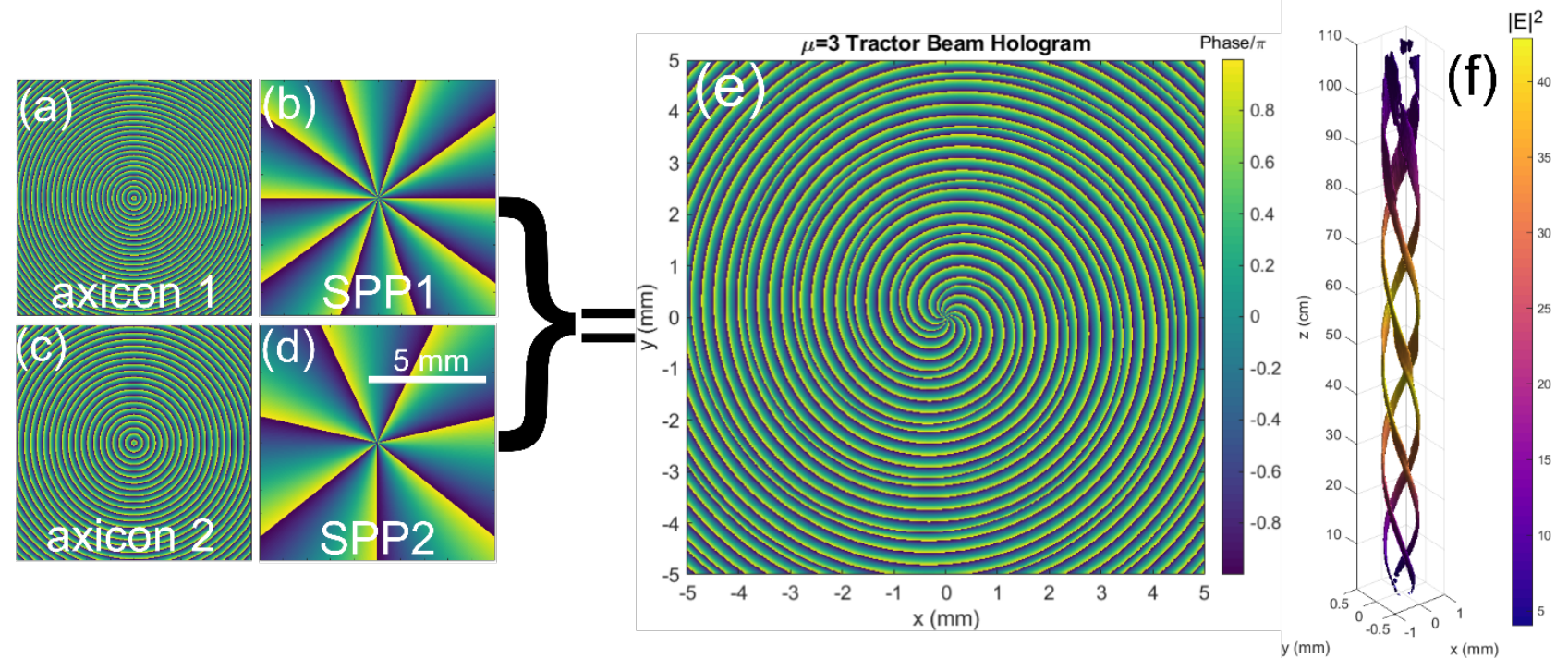

Fig. 1. Phase profiles of axicon lenses (a)\&(c) with internal angles 0.005 and 0.004 respectively and spiral phase plates (b)\&(d) with OAM of -10 and -7 respectively. The sum of these phase elements gives a tractor beam hologram (e), which can generate a non-diffracting solenoid beam (f).

A solenoid beam with $\mu$ in-plane radial intensity maxima can be formed from the coaxial superposition of two Bessel beams of order $m$ and $n$, with $|m-n|=\mu$. The Bessel beams can be generated from a Gaussian laser beam via axicon lenses used in conjunction with spiral phase plates (SPP) of OAM $m$ and $n$, or using their equivalent phase profiles. By using axicon phase profiles with the internal angles $\alpha$ and $\beta$, such that $n \cos (\alpha)>m \cos (\beta)$, it is possible for the solenoid beam to exert a net optical force on a Rayleigh scatterer in the retrograde direction, that is back towards the source, along a solenoidal path [2]. Choosing $\alpha$ and $\beta$ to take small values maximizes the length of the non-diffracting propagation region of the beam. The latter represents is the operating range of the tractor beam. Fig. 1a-d show the phase profiles of two axicon/SPP pairs designed to generate $m=-10, \alpha=0.005$ and $n=-7, \beta=0.004$ Bessel beams. Fig. 1e shows the weighted sum of these phase profiles, where the weighting is optimized to ensure the maxima 
of each Bessel beam spatially overlap [2]. This phase profile generates the solenoid beam isosurface shown in Fig. 1f where three intensity maxima orbit a $1 \mathrm{~mm}$ wide path and extend without diffraction for over $1 \mathrm{~m}$. The isosurface is calculated using scalar (Fresnel) diffraction, with the input light being a normally incident Gaussian beam with fullwidth-at-half-maximum (FWHM) of $6.6 \mathrm{~mm}$ and peak value of 1 , with phase modulated by the tractor beam hologram. The isosurface shows the $70 \%$ surface of the magnitude squared of the scalar field at each z-slice.
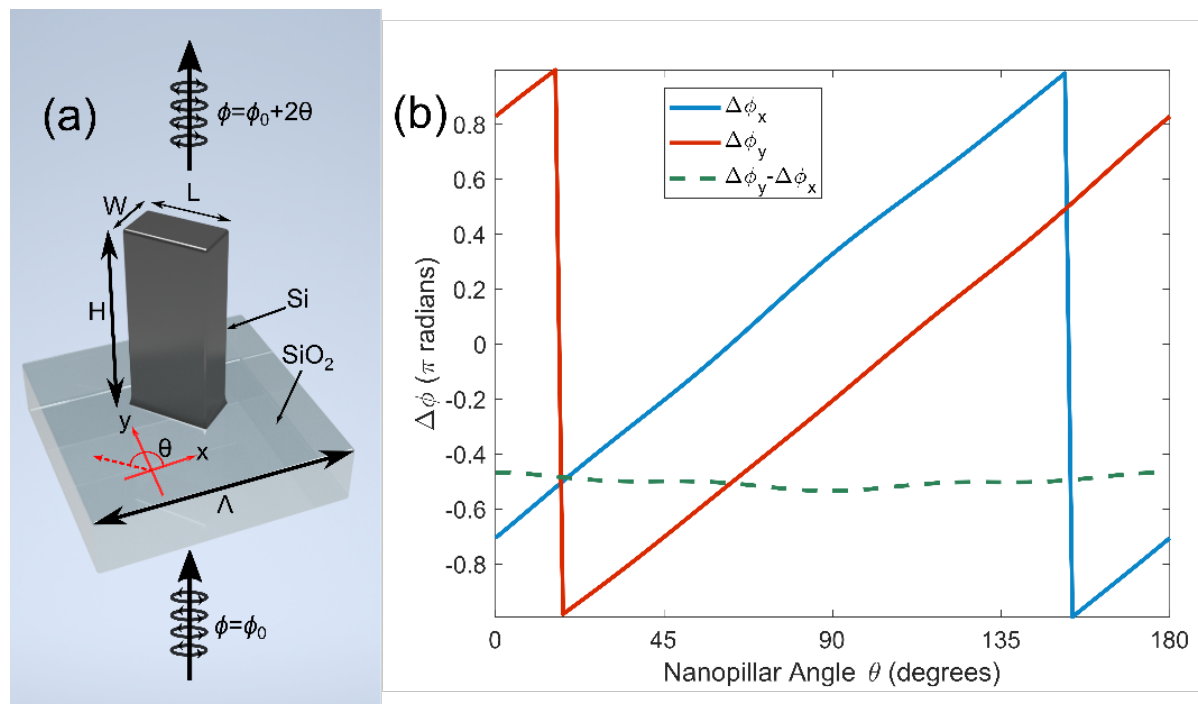

Fig. 2. (a) Schematic diagram of single Si element of metasurface hologram. (b) Calculated phase of $x$ (blue line) and y (red) components of the transmitted electric field as a function of nanopillar orientation. Phase difference between components is shown in green. Input beam is right circularly polarized (RCP).

We next use finite element modelling to design our tractor beam metasurface, i.e. to determine the geometric parameters of the silicon ( $\mathrm{Si}$ ) nanopillars on a glass substrate that comprise it. Fig. 2a shows a schematic of one such nanopillar, where a right circularly polarized beam (RCP) is converted to left circularly polarized (LCP) and the accumulated Berry phase is given by $2 \theta$, where $\theta$ is the angle the Si nanopillar long axis makes with the x-axis. At $\lambda=1064 \mathrm{~nm}$, with an array period $\Lambda=500 \mathrm{~nm}$, calculations show that a metasurface based on Si blocks with dimensions $(L, W, H)=(260 \mathrm{~nm}, 140 \mathrm{~nm}, 610 \mathrm{~nm})$ will tranmit over $98 \%$ of incident light. As seen in Fig. 2b, the phase difference between $\mathrm{y}$ - and $\mathrm{x}$-components of the beam exiting the metasurface is $-\pi / 2$, i.e. the metasurface converts RCP to LCP as desired. It can be furthermore seen from Fig. $2 b$ that the metasurface imparts an overall phase shift $\Delta \phi=2 \theta$, due to the accumulation of geometric (or Berry) phase. This confirms that our Si nanopillar approach can be employed to create the phase-only hologram in Fig. 1e, with one Si nanopillar for each pixel, with orientation relative to the $\mathrm{x}$-axis as given by $\theta=\Delta \phi / 2$. The metasurface can then be illuminated with a Gaussian laser beam (at $\lambda=1064 \mathrm{~nm}$ ) to generate the solenoidal tractor beam shown in Fig. 1f without the need for an SLM or bulk optics.

\section{Conclusion}

We have designed a silicon geometric phase metasurface hologram to generate a triple helix solenoid beam from a circularly polarized Gaussian laser beam input without the use of bulk optics such as an SLM or lenses. These types of non-diffracting beams have been shown to apply long-range retrograde (i.e. toward source) forces on microscopic objects. We anticipate that this capability will be very interesting for optical trapping, e.g. in microfluidic lab-on-achip applications for "pulling" such objects onto a sensor device for detection and identification.

\section{References}

[1] A. Mondal, A. Yevick, L.C. Blackburn, N. Kanellakopoulos, D.G. Grier, Projecting non-diffracting waves with intermediate-plane holography, Optics Express, 26 (2018) 3926-3931.

[2] A. Yevick, D.B. Ruffner, D.G. Grier, Tractor beams in the Rayleigh limit, Physical Review A, 93 (2016) 043807. 


\section{University Library}

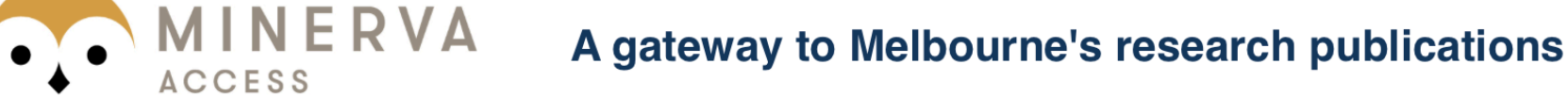

Minerva Access is the Institutional Repository of The University of Melbourne

Author/s:

Cadusch, J;Wen, D;Meng, J;Crozier, KB

Title:

Geometric Phase Metasurface Hologram for Optical Tractor Beam Generation

Date:

2020-01-01

Citation:

Cadusch, J., Wen, D., Meng, J. \& Crozier, K. B. (2020). Geometric Phase Metasurface Hologram for Optical Tractor Beam Generation. 2020 CONFERENCE ON LASERS AND ELECTRO-OPTICS (CLEO), Part F182-CLEO-QELS 2020, OSA \& IEEE. https:// doi.org/10.1364/CLEO_QELS.2020.FTu4Q.1.

Persistent Link:

http://hdl.handle.net/11343/294872 